\title{
Q.MAA
}

The Maximum Average Gain in a Sequence of Bernoulli Games

Author(s): Wolfgang Stadje

Source: The American Mathematical Monthly, Vol. 115, No. 10 (Dec., 2008), pp. 902-910

Published by: Mathematical Association of America

Stable URL: http://www.jstor.org/stable/27642637

Accessed: 25/02/2011 16:13

Your use of the JSTOR archive indicates your acceptance of JSTOR's Terms and Conditions of Use, available at http://www.jstor.org/page/info/about/policies/terms.jsp. JSTOR's Terms and Conditions of Use provides, in part, that unless you have obtained prior permission, you may not download an entire issue of a journal or multiple copies of articles, and you may use content in the JSTOR archive only for your personal, non-commercial use.

Please contact the publisher regarding any further use of this work. Publisher contact information may be obtained at http://www.jstor.org/action/showPublisher?publisherCode=maa.

Each copy of any part of a JSTOR transmission must contain the same copyright notice that appears on the screen or printed page of such transmission.

JSTOR is a not-for-profit service that helps scholars, researchers, and students discover, use, and build upon a wide range of content in a trusted digital archive. We use information technology and tools to increase productivity and facilitate new forms of scholarship. For more information about JSTOR, please contact support@jstor.org. 


\section{The Maximum Average Gain in a Sequence of Bernoulli Games}

\section{Wolfgang Stadje}

1. INTRODUCTION. Consider a sequence of games where one flips a (possibly biased) coin and wins $\$ 1$ if it comes up heads and loses $\$ 1$ if it comes up tails. At any time, one can compute the average gain for the games that have been played so far. What is the maximum average gain that will be achieved?

Formally, let $X_{1}, X_{2}, \ldots$ be independent Bernoulli random variables, indicating the successive outcomes and satisfying $\mathbb{P}\left(X_{i}=1\right)=p$ and $\mathbb{P}\left(X_{i}=-1\right)=q=1-p$ for some $p \in(0,1)$. Then $S_{n}=X_{1}+\cdots+X_{n}$ and $S_{n} / n$ are the total gain and the average gain, respectively, after $n$ games; we are interested in $M=\sup _{n \geq 1}\left(S_{n} / n\right)$. It is a classical result that the maximum total gain $\sup _{n \geq 1} S_{n}$ is almost surely equal to $\infty$ if the game is favorable to the player or fair, i.e., if $p \geq 1 / 2$; in the unfavorable case $p<1 / 2$ it is almost surely finite and its distribution is given by $\mathbb{P}\left(\sup _{n \geq 1} S_{n} \geq\right.$ $k)=(p / q)^{k}$ for all $k \in \mathbb{N}$ (see [6, Sect. XIV.2]). The maximum average gain $M$ is a much more complicated random variable. Besides being of mathematical interest, its consideration can be motivated as follows:

(a) Suppose that the player has to pay a participation fee of $c$ dollars for every game (if $c<0$ he receives this money to be enticed to play). Then what is the probability $\pi_{c}$ that he will at least once be on the winning side? Clearly, $\pi_{c}=\mathbb{P}\left(\sup _{n \geq 1}\left(S_{n}-n c\right)>\right.$ $0)=\mathbb{P}(M>c)$.

(b) Consider a baseball player and let $H_{n}$ be his number of hits after going to bat $n$ times. Then $B=\sup _{n \geq 1}\left(H_{n} / n\right)$ is his highest batting average ever attained. Assuming independent and identically distributed (i.i.d.) outcomes, we can reduce $B$ to $M$ by an affine transformation, defining $H_{n}=X_{1}^{\prime}+\cdots+X_{n}^{\prime}$ for the $\{0,1\}$-valued random variables $X_{i}^{\prime}=\left(X_{i}+1\right) / 2$; this leads to the relation $B=(M / 2)+(1 / 2)$. Of course, there are many other situations in which the random variable $B$ shows up as the maximum average number of successes observed in a long sequence of Bernoulli trials.

(c) In the theory of optimal stopping [2] one tries to terminate a sequence of random variables ("rewards") using some rule that does not depend on future events, so as to maximize the expected reward at the time of stopping. In particular, the maximization of $\mathbb{E}\left(S_{\tau} / \tau\right)$ over all stopping rules $\tau$ with respect to $\left(X_{n}\right)_{n \in \mathbb{N}}$ (i.e., rules $\tau$ such that for every $n$ the event $\{\tau=n\}$ can be defined in terms of $X_{1}, \ldots, X_{n}$ alone) has attracted a lot of attention (see, e.g., $[3,4]$ ). In the subarea of prophet inequalities, which deals with comparison of the maximum expected gain achievable by optimal stopping and the expected supremum of the underlying random sequence, the case of arithmetic means of i.i.d. random variables has not been solved, even for Bernoulli variables [8]. Neither $\sup _{\tau} \mathbb{E}\left(S_{\tau} / \tau\right)$ nor $\mathbb{E}(M)$ is known. This note may serve as an explanation for why computing $\mathbb{E}(M)$ is difficult.

A comprehensive account of simple random walks with steps \pm 1 was given in [6]. Already Pascal and Fermat have dealt with the classical gambler's ruin problem [5]; their solutions date back to 1656. The first published solution is due to Huygens. According to interpretation (a) above, our question can be considered as a variant of this 
problem by introducing fees or side payments into the game (and assuming that one player is infinitely rich).

Our first proposition shows an unusual feature of $M$ : it almost surely (a.s.) attains only rational values in the interval $(p-q, 1]$, and each of them with positive probability. There are hardly any other naturally occurring examples of random variables of this kind.

For this and the following results we need to refer to a few basic facts about random walks. Let $Y_{1}, Y_{2}, \ldots$ be i.i.d. random variables with finite mean and let $R_{n}=Y_{1}+$ $\cdots+Y_{n}, n \geq 1$.

(i) $R_{n} / n \rightarrow \mathbb{E}\left(Y_{1}\right)$ a.s. (strong law of large numbers [7, Sect. VII.8]).

(ii) If $\mathbb{E}\left(Y_{1}\right)=0$ and $Y_{1}$ is not a.s. equal to 0 , then $\limsup _{n \rightarrow \infty} R_{n}=\infty$ a.s. (case of zero drift [7, Sect. VI.10]).

(iii) If $\mathbb{E}\left(Y_{1}\right)<0$, then $\mathbb{P}\left(R_{n} \leq 0\right.$ for all $\left.n \geq 1\right)>0$ (case of negative drift [7, Sect. XII.2]).

We will also need the following fact, which was stated earlier, about the Bernoulli variables considered in this paper:

(iv) If $p<1 / 2$, then $\mathbb{P}\left(\sup _{n \geq 1} S_{n} \geq k\right)=(p / q)^{k}, k \geq 1$ [6, Sect. XIV.2].

\section{Proposition 1.}

$\mathbb{P}(M \in(p-q, 1] \cap \mathbb{Q})=1$ and $\mathbb{P}(M=x)>0$ for every $x \in(p-q, 1] \cap \mathbb{Q}$.

Proof. We have $S_{n} / n \leq 1$ for all $n \geq 1$ and $S_{n} / n \rightarrow p-q$ a.s. by (i). Further, $\left(S_{n}-(p-q) n\right)_{n \in \mathbb{N}}$ is a nontrivial random walk with mean zero so that, by (ii), limsup $\operatorname{sum}_{n \rightarrow \infty}\left(S_{n}-(p-q) n\right)=\infty$ a.s. It follows that $\mathbb{P}\left(S_{n} / n>p-q\right.$ for some $n \in \mathbb{N})=1$, yielding $p-q<M \leq 1$ a.s. Thus $\mathbb{P}(M \in(p-q, 1] \cap \mathbb{Q})=1$, since the supremum is a.s. attained.

Now let $r / s$ be some rational number in $(p-q, 1]$. To prove that $\mathbb{P}(M=r / s)>0$ we show that the following way to achieve $M=r / s$ has positive probability: The first $s-r$ outcomes are tails and are followed by $s+r$ heads; in particular, the average gain after $2 s$ steps is $r / s$ and this is the maximum so far. Given this event, the average gain will never exceed $r / s$ over the entire sequence if the average gain on the remaining steps after the first $2 s$ is always at most $r / s$, which in turn has positive probability by property (iii) above. This idea can be translated into the following computation:

$$
\begin{gathered}
\mathbb{P}(M=r / s) \geq \mathbb{P}\left(X_{i}=-1 \text { for } i=1, \ldots, s-r \text { and } X_{i}=1\right. \text { for } \\
\left.i=s-r+1, \ldots, 2 s \text { and } \frac{S_{2 s+n}}{2 s+n} \leq r / s \text { for all } n \geq 1\right) \\
=q^{s-r} p^{s+r} \mathbb{P}\left(S_{2 s+n}-S_{2 s} \leq(2 s+n) \frac{r}{s}-S_{2 s}\right. \\
\left.\quad \text { for all } n \geq 1 \mid S_{2 s}=2 r\right) \\
=q^{s-r} p^{s+r} \mathbb{P}\left(S_{n}-\frac{r}{s} n \leq 0 \text { for all } n \geq 1\right)
\end{gathered}
$$

and the right-hand side is positive by (iii), because $\left(S_{n}-(r / s) n\right)_{n \geq 1}$ is a random walk with negative drift (since $r / s>\mathbb{E}\left(X_{1}\right)$ ). Therefore, $\mathbb{P}(M=x)>0$ for every rational number $x \in(p-q, 1]$. 
The main aim of this paper is to derive explicit formulas for $\mathbb{P}(M \leq r / s)$ and $\mathbb{P}(M=r / s)$, where $r$ and $s$ are integers. We may assume that $s>0, r / s \in(p-q, 1]$, and that $r$ and $s$ are relatively prime. Two cases are simple: $r=s$ or $r=0$. We have

\section{Proposition 2.}

$$
\begin{aligned}
& \mathbb{P}(M=1)=p \\
& \mathbb{P}(M=0)=0, \quad \text { if } p \geq 1 / 2 \\
& \mathbb{P}(M=0)=\frac{p(q-p)}{1-p}, \quad \text { if } p<1 / 2 .
\end{aligned}
$$

Proof. First note that $\mathbb{P}(M=1)=\mathbb{P}\left(X_{1}=1\right)$ since $S_{n} / n<1$ for all $n \geq 2$ if $X_{1}=$ -1 and $S_{n} / n \rightarrow 2 p-1<1$. Further, if $p \geq 1 / 2,\left(S_{n}\right)_{n \geq 1}$ is a random walk with nonnegative drift so that $\lim _{\sup _{n \rightarrow \infty}} S_{n}=\infty$ by (i) and (ii), yielding (1.2). If $p<$ $1 / 2$, we can write $\mathbb{P}(M=0)=\sum_{n=1}^{\infty} \mathbb{P}\left(A_{n}\right)$, where $A_{n}$ is the event that the sequence $S_{1}, S_{2}, \ldots$ starts with $S_{1}=-1$ and visits zero exactly $n$ times, and every such visit is followed by a step downwards. If $p<q$, the probability of reaching zero from -1 is $p / q$ by (iv). Thus, in this case

$$
\mathbb{P}\left(A_{n}\right)=q[(p / q) q]^{n}\left(1-\frac{p}{q}\right)=p^{n}(q-p),
$$

and summing these probabilities yields (1.3).

From now on let $r \neq s$ and $r \neq 0$. To formulate our results, we need the roots of the complex polynomial

$$
f(z)=p z^{2 s}-z^{s+r}+q .
$$

We will show that $f(z)$ has only simple roots of which exactly $s+r$ have an absolute value less than or equal to 1 . Denote the roots by $z_{0}, z_{1}, \ldots, z_{2 s-1}$, where $z_{0}=1$, $\left|z_{i}\right| \leq 1$ for $i=1, \ldots, s+r-1$, and $\left|z_{i}\right|>1$ for $i=s+r, \ldots, 2 s-1$. If $s+r$ is even, let $z_{1}=-1$. It turns out that we can write $\mathbb{P}(M \leq r / s)$ and $\mathbb{P}(M=r / s)$ in terms of $z_{r+s}, \ldots, z_{2 s-1}$.

Theorem. Let $r, s \in \mathbb{Z} \backslash\{0\}, s>0, \operatorname{gcd}(r, s)=1$, and $r / s \in(p-q, 1)$. Then

$$
\mathbb{P}(M \leq r / s)=\prod_{i=r+s}^{2 s-1}\left(1-z_{i}^{-1}\right)
$$

and

$$
\mathbb{P}(M=r / s)=\prod_{i=r+s}^{2 s-1}\left(1-z_{i}^{-1}\right)+p \prod_{i=r+s}^{2 s-1}\left(1-z_{i}\right)
$$

Thus, to compute $\mathbb{P}(M \leq r / s)$ and/or $\mathbb{P}(M=r / s)$ one needs to find the roots of a polynomial of degree $2 s$. (Note that 1 or \pm 1 are among these roots, so the degree can immediately be diminished by 1 or 2.) The theorem is proved in Section 2. We give some explicit results in Section 3.

Before proceeding to the proof of the theorem it should be remarked that an alternative approach to the distribution of $M$ is provided by classical fluctuation theory, 
which leads to a complicated infinite series representation. From a famous theorem by Sparre Andersen (see [7, Sect. XII.7, Theorem 1]) we can conclude that

$$
\mathbb{P}(M \leq x)=\mathbb{P}\left(\sup _{n \in \mathbb{N}}\left(S_{n}-n x\right) \leq 0\right)=\exp \left\{-\sum_{n=1}^{\infty} \frac{1}{n} \mathbb{P}\left(S_{n}>n x\right)\right\} .
$$

For every $n$ the probability $\mathbb{P}\left(S_{n}>n x\right)$ can of course be expressed as a binomial sum.

2. PROOF OF THE THEOREM. We first have to consider the roots of the polynomial $f(z)=p z^{2 s}-z^{s+r}+q$. The only root of its derivative $f^{\prime}$ in $(0, \infty)$ is $c=$ $[(s+r) /(2 s p)]^{1 /(s-r)}$, and $c$ is larger than 1 since $r / s>2 p-1$. The reader can easily check that $f(0)=q>0, f$ is decreasing in a real neighborhood of 0 , and $f(1)=0$, $f^{\prime}(1)<0$. It follows that $f(c)<0$ and that $f$ is decreasing on $(0, c]$ and increasing to infinity on $[c, \infty)$. In particular, there is exactly one $b \in(c, \infty)$ such that $f(b)=0$, and we have $f(x)<0$ for all $x \in(1, b)$.

Let $K$ be a circle around 0 with radius strictly between 1 and $b$. Define $g(z)=$ $q+p z^{2 s}$ and $h(z)=-z^{s+r}$. If $z \in K$, then $0>f(|z|)=q+p|z|^{2 s}-|z|^{s+r}$ and thus

$$
|h(z)|=|z|^{s+r}>q+p|z|^{2 s} \geq\left|q+p z^{2 s}\right|=|g(z)| .
$$

By Rouché's theorem, $h(z)$ and $g(z)+h(z)=f(z)$ have the same number of roots in the interior of $K$ (each counted according to its multiplicity). As $K$ can be chosen arbitrarily close to either of the circles $\{z:|z|=b\}$ and $\{z:|z|=1\}$, there are exactly $s+r$ roots of $f$ having absolute value at most 1 , and no roots have absolute value in the interval $(1, b)$. Moreover, all roots of $f$ are simple because for $z \neq 0, f^{\prime}(z)=0$ implies that $|z|=c \in(1, b)$.

Consider a root $w_{0}$ of $f$ satisfying $\left|w_{0}\right|=b$ and $w_{0} \neq b$. Then

$$
\left|w_{0}\right|^{s+r}=\left|q+p w_{0}^{2 s}\right| \leq q+p\left|w_{0}\right|^{2 s}=q+p b^{2 s}=b^{s+r}=\left|w_{0}\right|^{s+r},
$$

so that $\left|q+p w_{0}^{2 s}\right|=q+p\left|w_{0}^{2 s}\right|$. This implies that $w_{0}^{2 s} \in(0, \infty)$ and then, as $f\left(w_{0}\right)=$ 0 , also $w_{0}^{s+r} \in(0, \infty)$. Let $m \in \mathbb{N}$ be minimal such that $w_{0}^{m} \in(0, \infty)$. Then $m \geq 2$, and $m$ is a common factor of $2 s$ and $s+r$, say $2 s=l m$ and $s+r=j m$ for some integers $l$ and $j$. If $s+r$ is odd, it follows from $\operatorname{gcd}(r, s)=1$ that $\operatorname{gcd}(2 s, s+r)=1$, and we arrive at a contradiction. Hence, in this case there is no $w_{0}$ as above, so $b$ is the only root of $f$ with absolute value $b$. Now let $s+r$ be even. Clearly $2 r=(2 j-l) m$, so $r$ and $s$ have the common factor $m / 2$ (if $m$ is even) or $m$ (if $m$ is odd). Thus if $m \geq 3$, we obtain $\operatorname{gcd}(r, s) \neq 1$, contradicting our assumptions. Therefore $m=2$, yielding $w_{0}=-b$.

Similar reasoning is possible for the roots satisfying $|z|=1$. We have proved:

Lemma. $f(z)$ has only simple roots. Besides $z=1$ there is exactly one more positive real root $b$, and $b \in(1, \infty)$. If $s+r$ is odd, $s+r-1$ of the roots have an absolute value strictly smaller than 1 and $s-r-1$ roots have an absolute value strictly larger than $b$. If $s+r$ is even, \pm 1 and $\pm b$ are the only real roots, $s+r-2$ of the roots have an absolute value strictly smaller than 1 , and $s-r-2$ roots have an absolute value strictly larger than $b$.

Proof of the Theorem. Fix $r$ and $s$ and define

$$
a_{m}=\mathbb{P}\left(S_{n} \leq \frac{r}{s} n+\frac{m}{s} \text { for all } n \in \mathbb{N}\right)
$$


Then $a_{0}=\mathbb{P}(M \leq r / s)$. Conditioning with respect to $X_{1}= \pm 1$ easily yields

$$
\begin{aligned}
& a_{m}=p a_{m+r-s}+q a_{m+r+s}, \quad m=s-r, s-r+1, \ldots \\
& a_{m}=q a_{m+r+s}, \quad m=0,1, \ldots, s-r-1 .
\end{aligned}
$$

Relations (2.1) and (2.2) can be written more compactly in the form of the following identity, which is valid for all $z \in \mathbb{C}$ for which $|z|<1$ :

$$
\sum_{m=0}^{s+r-1} a_{m} z^{m}=\frac{1}{q}\left(p z^{2 s}-z^{s+r}+q\right) \sum_{m=0}^{\infty} a_{m} z^{m} .
$$

From (2.3) we now derive explicit formulas for $a_{0}, \ldots, a_{s+r-1}$. We prove that

$$
\sum_{m=0}^{s+r-1} a_{m} z^{m}=\prod_{i=r+s}^{2 s-1}\left(1-\frac{1}{z_{i}}\right) \prod_{i=1}^{s+r-1}\left(1-\frac{z}{z_{i}}\right) .
$$

Recall that $z_{0}, \ldots, z_{2 s-1}$ are the (distinct) roots of $f(z)$, where $z_{1}, \ldots z_{s+r-1}$ have absolute value at most $1, z_{s+r}, \ldots, z_{2 s-1}$ have absolute value greater than 1 , and $z_{1}=-1$ if $s+r$ is even. Two cases have to be distinguished.

Case 1: $s+r$ is odd. By (2.3), the roots of $f(z)$ having absolute value smaller than 1 , that is, $z_{1}, \ldots, z_{s+r-1}$ are also roots of the polynomial $\sum_{m=0}^{s+r-1} a_{m} z^{m}$. Therefore

$$
\sum_{m=0}^{s+r-1} a_{m} z^{m}=a_{0} \prod_{i=1}^{s+r-1}\left(1-\frac{z}{z_{i}}\right) .
$$

To determine $a_{0}$, note that

$$
\frac{1}{q}\left(p z^{2 s}-z^{s+r}+q\right)=(1-z) \prod_{i=1}^{2 s-1}\left(1-\frac{z}{z_{i}}\right) .
$$

The random walk $\left(S_{n}-(r / s) n\right)_{n \geq 1}$ has a negative drift since $\mathbb{E}\left(S_{1}\right)-(r / s)=p-$ $q-(r / s)<0$, so that its supremum $T=\sup _{n \geq 1}\left(S_{n}-(r / s) n\right)$ is a.s. finite. As $a_{m}=$ $\mathbb{P}(T \leq m / s)$, it follows that $\lim _{m \rightarrow \infty} a_{m}=1$. Hence, replacing the complex $z$ by the real variable $x, \lim _{0<x \rightarrow 1^{-}}(1-x) \sum_{m=0}^{\infty} a_{m} x^{m}=1$ by Abel's limit theorem $[1, \mathrm{p} .42]$. Inserting (2.5) in (2.3) and letting $z$ run through a sequence of real numbers strictly increasing to 1 yields

$$
a_{0}=\prod_{i=r+s}^{2 s-1}\left(1-\frac{1}{z_{i}}\right)
$$

and (2.4) follows from (2.5).

Case 2: $s+r$ is even. The relation $\sum_{m=0}^{s+r-1} a_{m} z_{i}^{m}=0$ only holds for the roots inside the unit circle, i.e., for $i=2, \ldots, s+r-1$. (Recall that $z_{1}=-1$ in this case.) Hence there are constants $C_{1}$ and $C_{2}$ such that

$$
\sum_{m=0}^{s+r-1} a_{m} z^{m}=\left(C_{1} z+C_{2}\right) \prod_{i=2}^{s+r-1}\left(1-\frac{z}{z_{i}}\right) .
$$


Since $a_{m} \rightarrow 1^{-}$, it is easily seen that $\lim _{0>x \rightarrow-1^{+}}(1+x) \sum_{m=0}^{\infty} a_{m} x^{m}=0$. Thus, inserting (2.6) in (2.3) and letting $z$ tend to -1 through a decreasing sequence of real numbers yields $C_{1}=C_{2}$. It follows that $z_{1}=-1$ is also a root of $\sum_{m=0}^{s+r-1} a_{m} z^{m}$. Therefore, (2.5) is also valid if $s+r$ is even. The rest of the proof of (2.4) is the same as in Case 1.

Now we can compute the coefficients of the left-hand side of (2.4) by expanding the right-hand side:

$$
a_{m}=\sum_{1 \leq i_{1}<\cdots<i_{m} \leq s+r-1} \frac{(-1)^{m}}{z_{i_{1}} \cdots z_{i_{m}}} \prod_{i=r+s}^{2 s-1}\left(1-\frac{1}{z_{i}}\right), m=0, \ldots, s+r-1 .
$$

(1.4) is the special case $m=0$ of (2.7).

To prove (1.5), we proceed as follows:

$$
\begin{aligned}
\mathbb{P}\left(\sup _{n \geq 1}\left(S_{n} / n\right)<r / s\right) & =\mathbb{P}\left(S_{n} / n<r / s \text { for all } n \in \mathbb{N}\right) \\
& =\mathbb{P}\left(X_{1}=-1\right) \mathbb{P}\left(S_{n}-X_{1}<1+\frac{r n}{s} \text { for all } n \geq 2\right) \\
& =q \mathbb{P}\left(S_{n}<\frac{s+r}{s}+\frac{r n}{s} \text { for all } n \in \mathbb{N}\right) \\
& =q \mathbb{P}\left(s S_{n}<s+r+r n \text { for all } n \in \mathbb{N}\right) \\
& =q \mathbb{P}\left(s S_{n} \leq s+r+r n-1 \text { for all } n \in \mathbb{N}\right) \\
& =q \mathbb{P}\left(S_{n} \leq \frac{s+r-1}{s}+\frac{r n}{s} \text { for all } n \in \mathbb{N}\right) \\
& =q a_{r+s-1} .
\end{aligned}
$$

The first equality in (2.8) follows because $S_{n} / n \rightarrow p-q<r / s$ a.s., for the second one we condition on $X_{1}$, and for the third one we use that the sequence $S_{2}-X_{1}, S_{3}-$ $X_{1}, \ldots$ has the same distribution as $S_{1}, S_{2}, \ldots$ From (2.8) we obtain

$$
\mathbb{P}(M=r / s)=a_{0}-q a_{s+r-1}
$$

By (2.7) and the fact that $z_{1} \cdots z_{2 s-1}=q / p$, we get

$$
\begin{aligned}
a_{r+s-1} & =\frac{(-1)^{s+r-1}}{z_{1} \cdots z_{s+r-1}} \prod_{i=r+s}^{2 s-1}\left(1-\frac{1}{z_{i}}\right) \\
& =-\prod_{i=r+s}^{2 s-1}\left(1-z_{i}\right) / \prod_{i=1}^{2 s-1} z_{i} \\
& =-\frac{p}{q} \prod_{i=r+s}^{2 s-1}\left(1-z_{i}\right) .
\end{aligned}
$$

Now (1.5) follows immediately from (2.9) and (2.10). 
3. SOME EXPLICIT RESULTS. A determination of $z_{r+s}, \ldots, z_{2 s-1}$ in closed form in terms of radicals is possible for the fractions $r / s \in\{1 / 2,1 / 3,1 / 5,3 / 5\}$.

Example 1. $r=1, s=2$. Then

$$
f(z)=p z^{4}-z^{3}+q=(z-1)\left(p z^{3}-q z^{2}-q z-q\right) .
$$

Using Cardano's formulas [9, Ch. 8.8] for polynomial equations of third degree, we find that the only root outside the unit circle is

$$
\begin{gathered}
b=\frac{1-p}{3 p}+\frac{\sqrt[3]{-20 p^{3}+15 p^{2}+3 p+3 \sqrt{3} \sqrt{16 p^{6}-24 p^{5}+3 p^{4}+2 p^{3}+3 p^{2}}+2}}{3 \sqrt[3]{2} p} \\
-\frac{\sqrt[3]{2}\left(2 p^{2}-p-1\right)}{3 p \sqrt[3]{-20 p^{3}+15 p^{2}+3 p+3 \sqrt{3} \sqrt{16 p^{6}-24 p^{5}+3 p^{4}+2 p^{3}+3 p^{2}}+2}}
\end{gathered}
$$

We have for $p-q<1 / 2$, i.e., for $p<3 / 4$,

$$
P(M \leq 1 / 2)=1-b^{-1}
$$

and

$$
P(M=1 / 2)=1-b^{-1}-p(b-1) .
$$

For $p=1 / 2$ these probabilities are approximately 0.45631 and 0.0367 , respectively.

Example 2. $r=1, s=3$. Then

$$
f(z)=p z^{6}-z^{4}+q=\left(z^{2}-1\right)\left(p z^{4}-q z^{2}-q\right) .
$$

Thus one only has to solve a quadratic equation to obtain the roots; the ones outside the unit circle are

$$
\pm \sqrt{\frac{\sqrt{2 p+1-3 p^{2}}}{2 p}+\frac{1}{2 p}-\frac{1}{2}}
$$

The condition $p-q<r / s$ translates into $p \in[0,2 / 3)$, and for these values of $p$ we get

$$
\mathbb{P}(M \leq 1 / 3)=\frac{3}{2}-\frac{\sqrt{3 p+1}}{2 \sqrt{1-p}}, \quad \mathbb{P}(M=1 / 3)=\frac{2 p(\sqrt{(2-3 p) p+1}-1)}{\sqrt{(2-3 p) p+1}+1-p} .
$$

For $p=1 / 2$ we obtain $\mathbb{P}(M \leq 1 / 3)=(3-\sqrt{5}) / 2 \approx 0.38197$ and $\mathbb{P}(M=1 / 3)=$ $(7-\sqrt{5}) / 4 \approx 0.07295$.

Example 3. For $r / s=1 / 5$ and $r / s=3 / 5$ the corresponding polynomials are

$$
p z^{10}-z^{6}+q=\left(z^{2}-1\right)\left(p z^{8}+p z^{6}-q z^{4}-q z^{2}-q\right)
$$

and

$$
p z^{10}-z^{8}+q=\left(z^{2}-1\right)\left(p z^{8}-q z^{6}-q z^{4}-q z^{2}-q\right) .
$$


To find their roots one can set $w=z^{2}$ and solve polynomial equations of fourth degree. Of course, the resulting formulas are very long. For example, the real root $b>1$ of $p z^{10}-z^{8}+q($ case $r / s=3 / 5)$ is given by

$$
\begin{aligned}
b= & \frac{1}{2}\left[A(p)+\frac{B(p)}{p}+\frac{2 C(p)}{9 p B(p)}\right]^{1 / 2}+\left[A(p)-\frac{B(p)}{2 p}-\frac{C(p)}{9 p B(p)}\right. \\
& \left.\left.+D(p)\left[A(p)+\frac{B(p)}{p}+\frac{2 C(p)}{9 p B(p)}\right]\right]^{1 / 2}+\frac{1}{4 p}-\frac{1}{4}\right)^{1 / 2}
\end{aligned}
$$

where

$$
\begin{aligned}
& A(p)=\frac{(p-1)^{2}}{4 p^{2}}-\frac{2(p-1)}{3 p}, \\
& B(p)=\frac{\sqrt[3]{-25 p^{3}+30 p^{2}+15 p+3 \sqrt{3} \sqrt{-125 p^{6}+300 p^{5}-190 p^{4}+4 p^{3}+3 p^{2}-8 p+16}-20}}{3 \sqrt[3]{2}}, \\
& C(p)=5 p^{2}-4 p-1, \\
& D(p)=-\frac{(p-1)^{3}}{4 p^{3}}+\frac{(p-1)^{2}}{p^{2}}-\frac{2(p-1)}{p} .
\end{aligned}
$$

Since $\pm b$ are the only roots outside the unit circle, it follows that

$$
\mathbb{P}(M \leq 3 / 5)=1-b^{-1}, \quad \mathbb{P}(M=3 / 5)=1-b^{-1}-p(b-1)
$$

for $p<4 / 5$. In the case $p=1 / 2$ we obtain $b \approx 1.38827$.

For all other values of $r / s$ numerical methods seem to be required. Using Mathematica it is easy to compute $\mathbb{P}(M=r / s)$ and $\mathbb{P}(M \leq r / s)$ for any given values of $r$ and $s$. Let us write $\mathbb{P}_{p}(\cdot)$ to indicate the dependence on $p$. Clearly, $M$ is stochastically increasing in $p$, i.e., $p \mapsto \mathbb{P}_{p}(M \leq x)$ is decreasing on $[0,(x+1) / 2)$ for any fixed $x \in(-1,1)$. Note that $\lim _{x \nearrow 1} \mathbb{P}_{p}(M<x)=1-\mathbb{P}_{p}(M=1)=1-p$, and the limit seems to be approached quickly. For example, we have $\mathbb{P}_{2 / 5}(M \leq 3 / 4) \approx 0.5996$, $\mathbb{P}_{2 / 5}(M \in(3 / 4,1)) \approx 0.0004$ and $\mathbb{P}_{1 / 2}(M \leq 4 / 5) \approx 0.49951, \mathbb{P}_{1 / 2}(M \in(4 / 5,1)) \approx$ 0.00049 .

We can write $f(z)$ in the form

$$
f(z)=p\left(z^{2 s}-1\right)-\left(z^{s+r}-1\right),
$$

so that $f(z)$ is a simple linear combination of two cyclotomic polynomials. However, it is not clear whether its Galois group is solvable for any values of $p, r$, and $s$ other than the ones treated above.

ACKNOWLEDGMENT. I would like to thank two referees for their thoughtful comments and constructive suggestions that led to several improvements.

\section{REFERENCES}

1. L. Ahlfors, Complex Analysis, 2nd ed., McGraw Hill, New York, 1986.

2. Y. S. Chow, H. Robbins, and D. Siegmund, The Theory of Optimal Stoppping, Dover, New York, 1991; corrected reprint of 1 st ed. (1971). 
3. D. A. Darling, Contribution to the optimal stopping problem, Z. Wahrscheinlichkeitstheorie Verw. Geb. 70 (1985) 525-533.

4. - Convergence rates for iterative solutions to optimal stopping problems, in Adaptive Statistical Procedures and Related Topics, IMS Lecture Notes and Monographs Series 8 (1985) 18-28.

5. A. W. F. Edwards, Pascal's problem: The 'gambler's ruin,' Int. Stat. Rev. 51 (1983) 73-79.

6. W. Feller, An Introduction to Probability Theory and Its Applications, vol. 1, 3rd ed., Wiley, New York, 1968.

7. — - An Introduction to Probability Theory and Its Applications, vol. 2, 2nd ed., Wiley, New York, 1971.

8. T. P. Hill and R. P. Kertz, A survey of prophet inequalities in optimal stopping theory, Contemp. Math. 125 (1992) 191-207.

9. B. L. van der Waerden, Algebra, vol. 1, Springer, New York, 1991.

WOLFGANG STADJE works at the Department of Mathematics and Computer Science at the University of Osnabrück. He received most of his mathematical education at the University of Göttingen (Ph.D. 1978, habilitation 1981). His recent research interests are stochastic processes and their applications in operations research.

Department of Mathematics and Computer Science, University of Osnabrück, 49069 Osnabrück, Germany wolfgang@mathematik.uni-osnabrueck.de 